\title{
SERUM NEOPTERIN: A NEW NOVEL BIOMARKER IN ACUTE MYOCARDIAL INFARCTION
}

\author{
Srivatsan Elayalwar', Sasivathanam Natarajan², Jayaprada Lakshmipathi ${ }^{3}$ \\ ${ }^{1}$ Assistant Professor, Department of Biochemistry, KAPV Government Medical College, Periyamilaguparai, Trichy, Tamilnadu. \\ 2Professor, Department of Biochemistry, Thanjavur Medical College, Thanjavur, Tamilnadu. \\ ${ }_{3}^{3}$ Associate Professor, Department of Nutrition and Dietetics, Seethalakshmi Ramaswami College, Tiruchirappalli.
}

\section{ABSTRACT}

\section{BACKGROUND}

To elucidate the role of Neopterin levels and its short-term prognostic significance in AMI, the following objectives are formulated. (i) Estimation of levels of Serum Neopterin in AMI. (ii) Significance of levels of Serum Neopterin during the treatment in the ICCU. (iii) Correlation of levels of Serum Neopterin with the other cardiac risk factors. (iv) The role of Neopterin for the prognostic stratification in AMI.

\section{METHODS}

Patients admitted in ICCU with evidence of AMI are selected for the study.

\section{RESULTS}

After estimation of serum Neopterin in all these patients, it is found increased above normal and more increased in patients with MACE.

\section{CONCLUSION}

Studies have reported an association between Neopterin and the extent of atherosclerosis. Neopterin is elevated in patients with CAD and peripheral vascular disease. A central role in the pathogenesis of AMI is attributed to monocyte/macrophage activation. Neopterin, a pteridine derivative is synthesised by monocyte/macrophage upon stimulation with interferon. Neopterin levels are a useful tool to monitor monocyte/macrophage in AMI. Neopterin was identified as fluorescent component. It is clear that Neopterin can be detected in most body fluids. Inflammation plays a key role in the pathogenesis of atherosclerosis. Infiltration of neutrophils and monocytes/macrophages is detected in the vessel wall in patients with CAD. Macrophages activated by interferon gamma synthesise metalloproteinases and Neopterin, a pteridine derivative that has been used as an immunomarker. Neopterin as a marker of macrophage activation is significantly increased in patients with AMI, supporting the hypothesis of monocyte/macrophage activation in patients with AMI. Studies also found an association between Neopterin levels and impaired LVEF. Elevated serum levels of Neopterin, an immune modulator secreted by activated macrophages with patients in ACS. Serum Neopterin is an independent predictor of MACE in patients with ACS. This marker of macrophage may be useful for risk stratification in patients with AMI. The study proves that neopterin can be considered as a new novel biomarker for the disease activity in AMI and also as a prognostic marker for risk stratification in AMI to prevent mortality and MACE.

\section{KEYWORDS}

Serum Neopterin Acute Myocardial Infarction (AMI), Massive Adverse Cardiac Events (MACE) Diagnostic marker, Prognostic Marker, Left Ventricular Ejection Fraction (LVEF), Thrombolysis in Myocardial Infarction (TIMI).

HOW TO CITE THIS ARTICLE: Elayalwar S, Natarajan S, Lakshmipathi J. Serum neopterin: a new novel biomarker in acute myocardial infarction. J. Evolution Med. Dent. Sci. 2016;5(54):3582-3585, DOI: 10.14260/jemds/2016/826

\section{INTRODUCTION}

Acute Myocardial Infarction (AMI) is one of the most common diseases in hospitalised patients in industrialised countries. The early (30-day) mortality rate from AMI is $\sim 30 \%$ with more than half of these deaths occurring before the stricken individual reaches the hospital. Although, the mortality rate after admission for AMI declined by $\sim 30 \%$ over the past two decades. Approximately, 1 of every 25 patients who survives the initial hospitalisation dies in the first year after AMI.

Financial or Other, Competing Interest: None.

Submission 22-05-2016, Peer Review 21-06-2016,

Acceptance 27-06-2016, Published 05-07-2016.

Corresponding Author:

Dr. Srivatsan Elayalwar,

Assistant Professor,

Department of Biochemistry,

KAPV Government Medical College,

Periyamilaguparai, Trichy,

Tamilnadu, India.

E-mail: drsrivatsantrichy@gmail.com

DOI: $10.14260 /$ jemds $/ 2016 / 826$
Mortality is approximately four fold higher in elderly patients (Over age 75) compared with younger patients. AMI is one of the important medical emergencies admitted in the hospitals. It is characterised by the presence of clinical features like sudden onset of severe chest pain with dyspnoea, palpitation, and profuse sweating leading to cardiac arrhythmias, cardiogenic shock, cardiac failure, and death if not treated early. Among the biomarkers for diagnosis and prognosis in AMI, the Neopterin, a pteridine derivative is considered to be one of the new novel biomarkers, which is found to be elevated in patients with AMI ${ }^{1}$. Neopterin was first isolated from larvae of bee in 1963. Eventually, Neopterin was identified as the fluorescent component that was elevated in the urine of mice with Ehrlich ascites tumour. Now, it is proved that this is the exclusive product of monocytes/macrophages that have been stimulated by interferon gamma, a cytokine that is produced by activated T-lymphocytes and nature killer cells ${ }^{2}$. Biochemically, Neopterin belongs to pteridines group. It is a catabolic product of guanosine triphosphate [GTP]. 
Its molecular formula is $\mathrm{C}_{9} \mathrm{H}_{11} \mathrm{~N}_{5} \mathrm{O}_{4}$. Its molecular mass is 253.215. Neopterin is synthesised from GTP via GTP cyclohydrolase (GTP-CH). The activity of GTP-CH can be greatly enhanced by interferon gamma. 7,8 dihydroneopterintriphosphate $\left(\mathrm{NH}_{2} \mathrm{TP}\right)$ is on the biosynthetic pathway of 5,6,7,8 tetrahydrobiopterin $\left(\mathrm{BH}_{4}\right)$. $\mathrm{BH}_{4}$ represents the electron donor in the amino acid metabolic pathways. Human monocytes/macrophages lack the enzyme 6-pyruvoyltetra-hydro biopterin synthase, which converts $\mathrm{NH}_{2} \mathrm{TP}$ to 6pyruvoyl-tetra-hydro biopterin. Thus, in these cells, $\mathrm{NH}_{2} \mathrm{TP}$ accumulates and after hydrolysis by phosphatases is excreted as dihydroneopterin or Neopterin. Neopterin reflects the monocytes/macrophages activity stimulated by interferon gamma, a cytokine that is produced by the activated $\mathrm{T}$ lymphocytes the nature killer cells. Estimation of serum levels of Neopterin is a new novel biomarker in patients with acute and chronic coronary syndrome ${ }^{3}$. Atherosclerosis is a complex disease that involves lipoprotein influx and modification increased pro-oxidant stress and inflammatory angiogenic and fibroproliferative responses intermingled with extra cellular matrix and smooth muscle cell proliferation resulting in the formation of atherosclerotic plaque. The concept of response to injury, hypothesis considers atherosclerosis as a chronic inflammatory response of the arterial wall to the injured endothelium ${ }^{4}$. Inflammation plays important role in the pathogenesis of atherosclerosis. Atherosclerosis remains the major cause of death and premature disability in the developed countries ${ }^{5}$. Formation of fatty streak, the earliest phase of atherogenesis involves recruitment of leucocytes due to expression of leucocyte adhesion molecule on endothelial cells in turn triggered by primary proinflammatory cytokines. Subsequent migration of inflammatory cells into the subendothelial space requires chemotaxis controlled by chemokines induced by the primary cytokines. Mononuclear cells within this initial infiltrate as well as intrinsic vascular cells subsequently release growth factors that stimulate proliferation of smooth muscle cells and hence the progression of plaques ${ }^{6}$. Inflammation is a major feature of atherothrombosis and there is growing evidence of an association between systemic inflammation and the occurrence of stroke, peripheral artery disease, unstable angina, and IHD. Such an inflammatory component may be the final common result of a variety of infectious and noninfectious inflammatory stimuli and of the individual immunologic and inflammatory response. Triggers of plaque rupture and novel risk factors for ACS are: Neopterin, Lselectin, Macrophage inflammatory peptide, Interleukin, Intra Cellular Adhesion, Vascular Cell Adhesion, Leucocyte Count, Heat Shock Proteins, Matrix metalloproteinase 9 (MMMP 9). Inflammation plays a role in the pathogenesis IHD ${ }^{7}$. It is now accepted that inflammatory processes take place within the atheromatous lesions and significantly contribute to the progression of stenosis and acute coronary events ${ }^{8}$. Studies have shown an association between markers of inflammation and the presence of coronary atherosclerosis as well as the exacerbation of inflammation during acute myocardial ischemia. Several inflammatory markers have been shown to represent true cardiovascular risk factors. In this respect, the CRP, the Neopterin, and the Leucocyte count are all of particular interest. Studies suggest that high levels of Serum Neopterin may be a marker of atheromatous plaque vulnerability and identify patients at increased risk of serious coronary events 9 . The results of these studies reveal that Neopterin may be a marker of coronary disease activity rather than a marker of presence of CAD. Inflammation plays a vital role in atherosclerosis and coronary heart disease. Inflammatory processes of the coronary arterial wall are involved in plaque formation, progression, and finally plaque instability leading to clinical manifestations of stable coronary artery disease and acute coronary syndromes and heart failure. Biomarkers of inflammation emerged as potentially useful tools for risk stratification ${ }^{10}$. Elevated Serum levels of Neopterin, an immune modulator secreted by activated macrophages with patients in ACS. Serum Neopterin is an independent predictor of MACE in patients with ACS. This marker of macrophage may be useful for risk stratification and prognostic significance in patients with AMI11. Clinical studies performed recently proved great role of inflammatory processes in development of atherosclerosis. These inflammatory biomarkers are used in the diagnosis and identification of patients with unstable angina pectoris and myocardial infarct prediction of adverse cardiac events.(12)

\section{MATERIALS AND METHODS}

Neopterin is an activation marker for monocytes/macrophages and its circulating levels in the Serum is considered to be one of the prognostic markers in the treatment of AMI.

\section{Inclusion Criteria}

All cases admitted in the ICCU with the clinical features of AMI and with the positive documentation like increased cardiac enzyme levels, ECG findings, echo findings, etc. The group of patients in the age group between 45-65 years were selected irrespective of the gender difference. The associated findings like Hypertension, Diabetes Mellitus, and Hypercholesterolemia were also included in the study. Patients with acute symptoms only were selected irrespective of time of admission.

\section{Exclusion Criteria}

As the patients with features of tuberculous lesions, with features of atypical chest pain, with HIV infection, with the history of recent renal transplantation will also have high neopterin levels, which may interfere for the following study. The present study is about the serum neopterin levels in acute myocardial infarction only. Hence, the above-mentioned noncardiac diseases were excluded for the study, which may give false positive results. Apart from these patients with viral infection with autoimmune disease were also excluded for the study. The present study is the estimation of the serum Neopterin in patients with AMI and its short-term prognostic significance during hospitalisation. To start with the study, a protocol was framed with inclusion, exclusion criteria, aims and objectives, materials and methods, and with the review of literatures and references taken from various international journals and from the prescribed Text Books of Cardiology (Harrison's, Text Book of Medicine, Braunwald's Heart Disease). For this study, 40 patients were selected as study group and 40 patients were selected as control group based on the inclusion/exclusion criteria. The study group were collected from the ICCU Department of Cardiology. The blood samples of the study group were properly stored in the deep freezer after perfect centrifugation. The blood samples of the 
control group were collected from the normal persons and after perfect centrifugation and they were stored in the deep freezer. During collection, the usual precautions for venepuncture were observed. The grossly haemolytic, icteric, and lipemic samples were discarded. Samples were centrifuged again before assay to remove any particulate material. The Quantitative Estimation of Serum Neopterin was done using Neopterin ELISA, Enzyme-linked Immunosorbent Assay for the In-vitro Diagnostic Quantitative Determination of Serum Neopterin, obtained from IBL International.

\section{QUANTITATIVE DETERMINATION OF SERUM NEOPTERIN Test Principle}

Solid phase Enzyme-Linked Immunosorbent Assay (ELISA) based on the basic principle of a competitive ELISA ${ }^{13}$. An unknown amount of antigen in the sample and a fixed amount of enzyme-labelled antigen compete for the antibody binding sites (Rabbit-anti-neopterin). Both antigen-antibody complexes bind to the wells of the micro titre strips coated with a goat-anti-rabbit antibody. Unbound antigen is removed by washing. The intensity of the colour developed after the substrate incubation is inversely proportional to the amount of antigen in the sample.

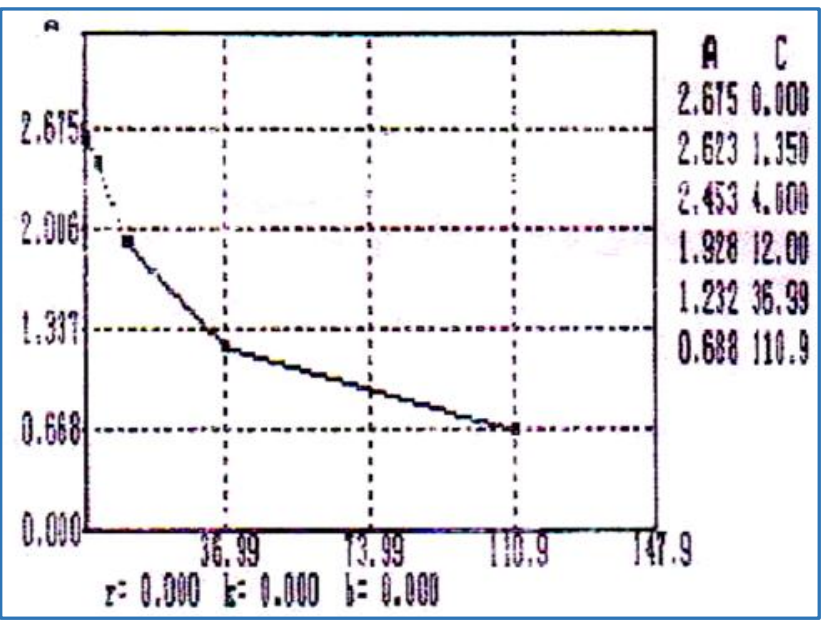

Quality Control for Serum Neopterin

\section{RESULTS}

The results obtained show that there is increase in neopterin level in AMI patients and there is no increase in control group. Statistically, there is highly significant association between the study group and levels of serum neopterin. $(\mathrm{P}<0.000<0.05$ value). Highly significant association between high levels of serum neopterin more than $25 \mathrm{~nm} /$ litre and MACE $(\mathrm{P}<0.000$ $<0.05$ value). Highly significant association between high levels of serum neopterin and history of previous hospitalisation for the same episode obtained. $(\mathrm{P}<0.000<0.05$ value). Statistically, there is highly significant association between high levels of serum neopterin and occurrence of death. $(\mathrm{P}<0.000<0.05$ value $)$.

\begin{tabular}{|c|c|c|c|}
\hline Parameters & $\begin{array}{c}\text { Serum } \\
\text { Levels of } \\
\text { Neopterin }\end{array}$ & $\begin{array}{c}\text { Statistical } \\
\text { Significance }\end{array}$ & P Value \\
\hline $\begin{array}{c}\text { Previous history } \\
\text { of hospitalisation } \\
\text { with features } \\
\text { of AMI }\end{array}$ & High & Significant & $\begin{array}{c}(\mathrm{P}<0.000 \\
<0.05 \\
\text { value })\end{array}$ \\
\hline
\end{tabular}

\begin{tabular}{|c|c|c|c|}
\hline AMI & High & Significant & $\begin{array}{c}(\mathrm{P}<0.000 \\
<0.05 \\
\text { value })\end{array}$ \\
\hline MACE & Very High & Significant & $\begin{array}{c}(\mathrm{P}<0.000 \\
<0.05 \\
\text { value })\end{array}$ \\
\hline Death & Very High & Significant & $\begin{array}{c}(\mathrm{P}<0.000 \\
<0.05 \\
\text { value })\end{array}$ \\
\hline
\end{tabular}

AMI-Acute Myocardial Infarction.

MACE-Massive Adverse Cardiac Events.

\section{DISCUSSION}

The present study was undertaken in patients who were admitted with clinical features of AMI in ICCU. The patients were in the age group of between 45-65 years including both genders with or without associated risk factors like Hypertension, Diabetes Mellitus, Hypercholesterolemia, etc. The levels of Serum Neopterin were estimated both in the study group and the control group and the levels were statistically correlated with other biochemical factors and clinical features. Serum Neopterin levels were more than normal in the entire study group, which signifies that Serum Neopterin levels were increased in AMI. The statistical correlation between the high levels of Neopterin and AMI was found to be highly significant [P $(0.000)$ at 0.01 level 2 tailed] In contrast, Serum Neopterin level were found to be within normal limits in the control group, which signifies that there is no disease activity in this group. The high Serum Neopterin levels were associated with Massive Adverse Cardiac Events (MACE) like Cardiac Arrhythmias, Cardiac Blocks, Cardiogenic Shock, Cardiac Failure, etc. The correlation between the higher levels of Serum Neopterin more than $25 \mathrm{nmol} /$ litre and the occurrence of MACE in the patients was statistically significant [P (0.000) at 0.01 level 2 tailed]. Hence, Neopterin can be considered not only as a biomarker for disease activity, but also a prognostic marker to identify the high risk patients during treatment and to implement emergency and essential medical intervention to prevent mortality. The study group with high Serum Neopterin levels were followed up critically during their hospitalisation and it is evident from the study that the occurrence of MACE and mortality is highly significant [P (0.000) at 0.01 level 2 tailed]. According to the above study, it is proved that serum levels of Neopterin can be considered as an independent biomarker for the disease activity and for the prognostic activity in AMI. There is correlation between Serum Neopterin more than $25 \mathrm{nmol} /$ lit and with previous history of hospitalisation and there is statistically significant correlation $[\mathrm{P}(0.000)$ at 0.01 level 2 tailed]. There is significant correlation with high levels of Serum Neopterin more than 25 and the occurrence of death in patients with AMI. The studies reviewed have demonstrated that Neopterin level is an important predictor of future cardiac as well as vascular adverse event. In particular, Neopterin levels predict future major cardiac and vascular adverse events in patients presenting with chronic coronary artery disease with acute coronary syndromes. In those with many of the above cited studies, underline the strong association between high Neopterin levels and complex atherosclerotic lesions, but not with disease extension giving account for the prognostic properties of Neopterin. This renders this molecule a useful marker of atherosclerotic plaque activity permitting the 
identification of the subjects at highest risk for MACE. Taken together, observations from all the studies reviewed propose the existence of a strong link between high Neopterin levels and cardiovascular risk profile suggesting a potential clinical use of Neopterin as a marker for disease activity in subjects with cardiovascular disease. This could help in identifying patients who are at a higher risk of developing cardiovascular adverse events who might benefit from urgent preventive strategies exploitation or extensive diagnostic workup as well as actual therapy depending from their comorbidities.

\section{CONCLUSION}

In this study, it is proved that monocyte/macrophage activation in AMI is reflected by the levels of Neopterin and a strong correlation was found between high levels of Neopterin and MACE. To conclude, Neopterin concentrations are usually increased in AMI. Highly-elevated Neopterin concentrations are among the best predictors of adverse outcome in patients with AMI risk. Braunwald's had initially proposed risk stratification based on the History, Physical examination, and Electrocardiogram. The American college of Cardiology/ American Heart Association Guidelines Committee later refined and incorporated cardiac markers for risk stratification. This study postulates that Neopterin can be considered as a new novel biomarker for the disease activity in AMI and also as a prognostic marker for risk stratification in AMI to prevent mortality and MACE.

\section{REFERENCES}

1. Gupta S, Fredericks S, Schwartzman RA. Serum neopterin in acute coronary syndromes. Lancet 1997;349:1252-3.

2. Alber HF, Duftner C, Wanitschek M, et al. Neopterin, CD4+CD28-lymphocytes and the extent and severity of coronary artery disease. International Journal of Cardiology 2009;135(1):27-35.

3. Schumacher M, Halwachs G, Tatzber F, et al. Increased neopterin in patients with chronic and acute coronary syndromes. Journal of the American College of Cardiology 1997;30(3):703-7.
4. Tatzber F, Rabl H, Koriska K, et al. Elevated serum neopterin levels in atherosclerosis. Atherosclerosis 1991;89(2-3):203-8.

5. Weiss G, Willeit J, Kiechl S, et al. Increased concentrations of neopterin in carotid atherosclerosis. Atherosclerosis 1994;106(2):263-71.

6. Schumacher M, Eber B, Tatzber F, et al. Neopterin levels in patients with coronary artery disease. Atherosclerosis 1992;94(1):87-8.

7. Fuster V, Badimon L, Badimon JJ, et al. Mechanisms of disease. I-the pathogenesis of coronary artery disease and the acute coronary syndromes. $N$ Engl J Med 1992;326(4):242-50.

8. Garcia-Moll X, Coccolo F, Cole D, et al. Serum neopterin and complex stenosis morphology in patients with unstable angina. Journal of the American College of Cardiology 2000;35(4):956-62.

9. Hermus L, Schuitemaker JHN, Tio RA, et al. Novel serum biomarkers in carotid artery stenosis: useful to identify the vulnerable plaque ? Clinical Biochemistry 2011;44(16):1292-8.

10. Sasaki T, Takeishi Y, Suzuki S, et al. High serum level of neopterin is a risk factor of patients with heart failure. International Journal of Cardiology 2010;145(2):318.

11. Auer J, Berent R, Lassnig E, et al. Prognostic significance of immune activation after acute coronary syndromes. Journal of the American College of Cardiology 2002;39(11):1878.

12. Avanzas P, Arroyo-Espliguero R, Quiles J, et al. Elevated serum neopterin predicts future adverse cardiac events in patients with chronic stable angina pectoris. European Heart Journal 2005;26:457-63.

13. Glynn LE. Neopterin. Biochemistry-methods-clinical application. In: Wachter H, Fuchs D, Hausen A, et al. (eds.) Cell biochemistry and function. Berlin and New York: Walter de Gruyter 1992. 